\title{
Principios metafísicos fundamentales de la cosmología abyayalense
}

\author{
Fundamental metaphysical principles of the \\ abyayalense cosmology
}

\author{
Juan Camilo Hernandez Rodriguez \\ Universidad Pedagógica Nacional (Bogotá, Colômbia) \\ Orcid 0000-0001-6675-3636 \\ juancamilohernandezrodriguez@gmail.com
}

Resumen: En el presente texto se hará una exposición general de los principales principios metafísicos que fundamentan la cosmología abyayalense colombiana. Para cumplir tal cometido se realizarán los siguientes pasos: primero, plantear el tipo de acercamiento filosófico que se puede establecer con la cosmología abyayalense; segundo, identificar los compromisos ontológicos de dicha cosmología, identificándola como monista (la realidad-naturaleza es una), pero internamente múltiple y en devenir; tercero, elucidar qué tipo de relaciones fundamentales permiten que se consoliden estructuras cósmicas (es decir, de «ordenamiento»), tanto a nivel macro como a nivel micro, a partir del concepto 'ley de origen' (en arhuaco, kunsamu; en wiwa, shembuta); y, finalmente, esbozar la idea del chamán como aquel ser que transita por esa compleja estructura cósmica para proteger el territorio.

Palabras clave: filosofía amerindia, metafísica indígena, ley de origen, ontología inmanente, visión holográfica. 
Abstract: In this paper we do a general exposition on the main metaphysical principles that underlie Colombian abyayalense cosmology. In order to fulfill this objective, the paper will carry the following steps: first, we propose the kind of philosophical approach that can be established in the abyayalense cosmology. Second, we will trace the ontological commitments of this cosmology, identifying it as monistic (reality and nature are one), but internally multiple and in becoming. Third, to elucidate what kind of fundamental relationships allow cosmic structures (that is to say "an ordering") to be consolidated, both at the macro and micro levels, from the concept of 'law of origin' (in arhuaco, kunsamu; in wiwa, shembuta). Finally, we outline the idea of the shaman as a being who go through that complex cosmic structure to protect the territory.

Key words: amerindian philosophy, indigenous metaphysics, Origin Law, immanent ontology, holographic view.

\section{Introducción}

Si tenemos en cuenta los esfuerzos actuales de la filosofía intercultural por comprender del pensamiento filosófico amerindio, ${ }^{1}$ podremos observar prolijos trabajos acerca de la metafísica náhuatl (León Portilla, 2017; Maffie, 2014; Hernández, 2019), maya (Mata Gavidia, 1950; Lenkersdorf, 2003; 2005; De la Garza, 1987; León-Portilla, 1994) e incaica —también llamada «andina»- (Estermann, 2009; Mariátegui, 2007; Yáñez del Pozo, 2002; Palacios, 2019). Varios de ellos incluso han investigado el chamanismo, el consumo de plantas enteógenas y su rol en su cosmología (De la Garza, 2012; Schultes, Hofmann y Rälsch, 2000; Ochoa, 2002). Sin embargo, no sucede lo mismo si nos preguntamos por la metafísica abyayalense ${ }^{2}$ de las comunidades indígenas del actual territorio colombiano (arhuacos, wiwa, em-

$1 \quad$ Agradezco profundamente a mi maestra, Consuelo Pabón Alvarado, $-\mathrm{y}$, junto con ella, a sus maestros, los mayores - por todas sus orientaciones y ayudas para la elaboración de este texto. Espero que él pueda ser de utilidad para la comunidad filosófica en su acercamiento a las filosofías amerindias.

2 «En tule, la lengua de los kunas, Abya-Yala quiere decir 'tierra-en-plena-madurez', fórmula totalmente en contravía de Nuevo Mundo, impuesta por quienes tenían interés de fraguarlo a su ambicioso acomodo» (Urbina y Peña, 2016, pp. 8-9). 
bera, kogui, uitoto, gunadule, etc.). Gran parte de este desinterés por el pensamiento indígena colombiano puede evidenciarse en que son muy pocos los estudios que intentan abordar de manera filosófica — no solamente antropológica - el pensamiento indígena abyayalense; siendo una excepción Páramo (2004), Urbina (2004) o Pabón (1993; 2002), o bien: los grupos de investigación Asterión y Merawi, de la Universidad Nacional de Colombia y la Universidad Pedagógica Nacional de Colombia, respectivamente (de los cuales esos tres investigadores han sido partícipes y gestores, según corresponda). ${ }^{3}$

Teniendo en cuenta esta carencia, se hace manifiesta la necesidad de realizar más estudios que eluciden la cosmovisión indígena colombiana desde una perspectiva filosófica. Por eso, en el presente texto me propondré elucidar desde la metafísica los principios fundamentales de la cosmología abyayalense. Sostendré que la cosmología abyayalense tiende a una visión inmanente de la realidad: no hay más que la naturaleza. Sin embargo, esta naturaleza internamente contiene múltiples dimensiones; cada una de ellas interconectadas ontológicamente, pero no necesariamente perceptibles. Sostendré también que el fundamento integrador de todas esas dimensiones espacio-temporales de la realidad es la ley de origen. Además, que gracias a esta fundamentación e integración de esos elementos de forma armónica hay una integración isomórfica entre micro y macrocosmos, también denominada: visión holográfica. No obstante, antes de realizar la explicación de estos tres principios cosmológicos aclararé primero desde qué propuesta metodológica asumiré la discusión y por qué no debería ser problemática la existencia de contenidos religiosos en el desarrollo de un pensamiento filosófico.

\section{La cosmología: el puente metafísico entre ciencia y religión}

Podría decirse que una razón principal de esa reserva y resistencia por parte de las comunidades filosóficas occidenta-

3 Si bien aquí se mencionan algunos notables esfuerzos de esos investigadores, el lector reconocerá que los esfuerzos siguen siendo escasos; tres investigadores y dos grupos de investigación (de hecho, desde hace varios años inactivos) no son suficientes para que la difusión de toda una tradición filosófica sea efectiva... 
les por reconocer elementos filosóficos valiosos en dichas culturas indígenas - ya no solo la abyayalense, sino también la mexica, incaica y maya- se debe al estrecho vínculo que tienen esos aspectos filosóficos con lo mitológico; de ahí que a veces se sugiera hablar de «pensamiento», «sabiduría» o «religión», pero no de «filosofía» (Beorlegui, 2010, p. 81). Este prejuicio de que lo religioso es un obstáculo para la filosofía podemos, cuando menos, retrotraerlo al siglo XIX, donde nos dice Eduard Zeller (1968):

No todo pueblo ni siquiera cada comunidad civilizada ha tenido filosofía. Muchos pueblos poseen santos, profetas y reformadores religiosos, mas sólo muy pocos han producido filósofos. Entre los pueblos de la antigüedad, aparte de Grecia, únicamente China e India se hallan en tal situación. (p. 10).

No obstante, justo después de decir esto, incluso descarta esas dos tradiciones o las considera a inferiores a las griegas porque

Fue este equilibrio, combinado con un vigoroso sentido de la realidad y un idéntico poder de abstracción el que los habilitó desde muy temprano para reconocer sus ideas religiosas según lo que ellas realmente eran — creaciones de la imaginación artística- y colocar en lugar de un mundo mitológico otro de ideas construido por la energía del pensar humano independiente, el Logos, el que podía pretender explicar la realidad de modo natural. (p. 11).

Grosso modo, el argumento de este y otros muchos autores es que la filosofía acontece cuando se abandona lo religioso (el $\mu \tilde{v} \theta$ os) para adentrarse en lo racional (el $\lambda$ ó $\gamma$ $\varsigma$ ). No obstante, que la filosofía tenga vínculos con lo religioso no debería ser obstáculo para que allí, en medio de lo simbólico y mítico, se encuentren elementos abstractos con alta carga metafísica y filosófica (de ahí que el filósofo europeo no titubee en reconocer que diversas obras de Platón, san Agustín y santo Tomás son filosóficas). Esta actitud general — que no ha sido problema solo de la filosofía amerindia, sino también de las filosofías india, china, japonesa y africana - se torna inadecuada y un tanto «condescendiente» (por no decir «hipócrita»). De hecho, puede evidenciarse en el extenso estudio de Kathryn Morgan (2004) 
que el mito nunca desapareció entre los filósofos griegos, sino que hubo, por así decirlo, una logización del mito":

[...] los dos polos podrían penetrarse entre sí [...] El mito no es totalmente irracional; la filosofía (al menos antes de Aristóteles) no está totalmente decantada de elementos míticos. (p. 32).

Luego, no es tendría por qué ser descabellado hablar de filosofías amerindias por el hecho de que elementos religiosos o mitológicos sean el punto de partida de la reflexión. Puede suceder, por ejemplo, que del mito o el relato religioso se abstraigan conceptos, estructuras y cuestionamientos altamente filosóficos. Algo de esto nos dice Urbina (2004):

Él [un abuelo indígena] era capaz de desmontar el cuento parcial, para ver sus estructuras y mirar que esos cuentos en el fondo estaban diciendo lo mismo. Esto es ya caminar en la dirección de la filosofía en el sentido estricto. [...] Hay un código estricto, una lógica interna que subyace a las apariencias que muestran diversidades y hasta contradicciones. Estos metacódigos míticos son los que perciben y llegan a manejar algunos sabedores indígenas, muy pocos, por cierto, como son bien los pocos verdaderos filósofos de Occidente. (p. 144).

Pues, bien, en concordancia con lo anterior, un recurso al que pretendo recurrir es el del concepto de 'cosmología' como un puente filosófico entre la ciencia y la religión. Tanto la religión como la ciencia poseen un área de su saber denominada con este nombre ('cosmología'). Igual sucede con la filosofía, siendo la cosmología una rama de la metafísica encargada de preguntas acerca de la estructura fundamental de la realidad. ${ }^{5}$ Cada una

$4 \quad$ Incluso, ya lo decía el mismo Jaeger (2001) en su Paideia: «El comienzo de la filosofía científica no coincide, así, ni con el principio del pensamiento racional ni con el fin del pensamiento mítico. Auténtica mitogonía hallamos todavía en el centro de la filosofía de Platón y de Aristóteles. Así, en el mito del alma de Platón o en la concepción aristotélica del amor de las cosas por el motor inmóvil del mundo» (p. 151).

5 Téngase en cuenta que la metafísica (el estudio de la realidad y sus principios) se compone de diversas ramas, cada una con un problema y posturas distinguibles: mereología (el estudio de los compuestos; relación todo-partes), usología (el estudio de las esencias), aitiología (el estudio de las causas y la posibilidad de la causación), teología (el estudio de Dios [impersonal] como fundamento metafísico) y cosmología (el estudio del ordenamiento de la realidad), a veces asociada al grounding (Sider, Hawthorne y Zimmerman, 2008). 
de estas cosmologías, a su manera y con métodos distintos, pretenden dar cuenta del origen, ordenamiento — sobre todo, este

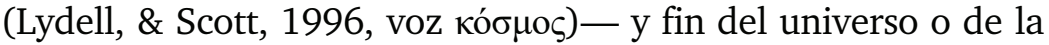
realidad en su conjunto. Por una parte, como lo sostuvo Quine (1984) en su momento, toda teoría científica tiene un compromiso ontológico con la cual se compromete y, en virtud de este, procura realizar la mejor explicación posible de la realidad. De otro lado, como bien lo muestra Keiji Nishitani (1999),

Cada religión, cuando adopta su forma concreta - como una realidad histórica efectiva-, siempre se basa en alguna visión del mundo u ontología. Para esta religión esta filosofía básica no es algo que pueda ser cambiado a voluntad, como un traje, sino que es como el agua al pez: una condición esencial para la vida. (p. 129 [cursivas fuera del texto]).

En este sentido, ese compromiso ontológico de cada cosmología nos permitiría concluir que «A decir verdad, la metafísica y la filosofía han consistido desde la antigüedad en la exploración dela línea divisoria entre la ciencia y la religión» (p. 129); o diríamos mejor, consistiría en: aquel campo común en el que la cosmología metafísica permite transversalizar y, además, profundizar en los fundamentos abstractos que están implícitos en esos compromisos ontológicos. En este sentido, la cosmología metafísica podría operar como un estudio de segundo orden desde el cual se indagan los principios filosóficos implícitos en la cosmovisión indígena: sus compromisos ontológicos, las relaciones de dependencia entre esas entidades, la estructuración de la realidad que se realiza a partir de ambas, etc. ${ }^{6}$ Luego, podemos, como lo indica el título de este texto, hablar de «Principios metafísicos fundamentales de la cosmología chamánica abyayalense».7

Desde esta propuesta, «La metafísica se asume, pues, como un estudio de segundo orden en el que se desarrollan sistemas teóricos que pueden fungir como modelos para que aquellas teorías que pretendan ofrecer explicaciones acerca de cómo es el mundo puedan acogerse o no a las implicaciones que tiene aceptar tal o cual tesis (realismo, antirrealismo, idealismo, materialismo, monismo, pluralismo, etc.)» (Hernández, 2020a, p. 139). Véase en el texto citado un mayor desarrollo del porqué y el cómo entender la metafísica de esta manera.

$7 \quad$ Otra forma de resolver la cuestión de si en el pensamiento indígena puede o no haber filosofía, podría ser comprender el filosofar del indígena desde concepción de Hadot (2009): la filosofía como un modo de vida. Esta reflexión o paradigma podría dar la pauta para que futuras investigaciones sobre la filosofía amerindia se desarrollen sin tantos atavíos... 
Así, eludiendo un poco discusiones a las que suelen llevar estos debates en filosofía intercultural acerca de cuál es la definición correcta de 'filosofía' — claro está, con criterios objetivos y que no sean reduccionistas-, este recurso de la cosmología metafísica como puente entre lo mítico y lo científico o «racional» podría permitirnos comprender de una mejor manera si hay o no elementos filosóficos en la cosmovisión abyayalense.

\section{Dualidades no disyuntivas: cuerpo energético y cuerpo material}

Lo primero que se debe tener en cuenta para comprender la cosmología chamánica es que esta es de carácter inmanente. Esto quiere decir que, a diferencia de las metafísicas trascendentes, en esta cosmovisión los principios de la realidad son intrínsecos a la naturaleza o el mundo (Hernández, 2019, p. 252). Luego, en estas cosmovisiones carece de sentido hablar de algo sobrenatural. La realidad es, pues, una y autosuficiente (monismo). Por lo mismo, al no haber algo trascendente (en el sentido radical de la palabra ya mencionado) se sostiene que todo existe y, por lo tanto, todo es real.

Este punto — «todo existe y todo es real»— ha sido objeto de debate, no solo de metafísica —el problema del no-ser o las apariencias-, sino también de la metaontología —¿qué significa 'existir' y 'ser real'?- (Berto y Plebanny, 2015, pp. 15-122). Un ejemplo de este problema lo podemos ver en los griegos, quienes también discutieron esas tesis («todo existe» $\mathrm{y}$ «todo es real»), asumiendo posturas diversas. ${ }^{8}$ Sostener que todo es real (en el sentido de «auténtico, verdadero, efectivo») conllevaría aceptar que los sueños o imaginarios también hacen parte del mundo y, por lo tanto, existen. Por su parte, en la cosmología chamánica abyayalense, tanto los sueños como las

\footnotetext{
$8 \quad$ Un interesante vínculo entre los griegos y el chamanismo puede evidenciarse en el trabajo de Magoja (2018), titulado: Sócrates: entre filosofía y chamanismo. Véase también un rastreo del debate acerca de la corresponden-

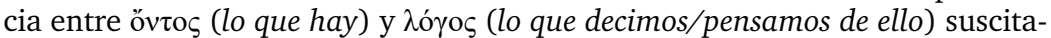
do en los griegos por este compromiso ontológico acerca de las implicaciones de asumir o rechazar el panrealismo en: Hernández (2020b).
} 
visiones generadas por los enteógenos son consideradas como auténticas y reales (Reichel-Dolmatoff, 2005, p. 24).

En este orden de ideas, no es apropiado hablar de 'representaciones', 'apariencias', 'imaginarios' o 'ilusiones' en el sentido de afirmar que «hay algo que parece real, pero en realidad no existe». ${ }^{9}$ Así, la cosmología abyayalense asume un pluralismo bastante rico y amplio en el compromiso ontológico de su cosmología: hay elementos, rocas, plantas, animales, monstruos, planetas y dioses; todos ellos se consideran, no solo entidades actuales, sino también seres vivos (pananimismo). Dos ejemplos de esa rica pluralidad de entidades y su interacción con los humanos los podemos encontrar en los siguientes pictogramas ubicados en el Chiribiquete y la Lindosa, respectivamente:

Ilustración 1. Pictogramas del Chiribiquete y la Lindosa que reflejan la pluralidad diversa de entidades asumidas en la cosmovisión abyayalense.
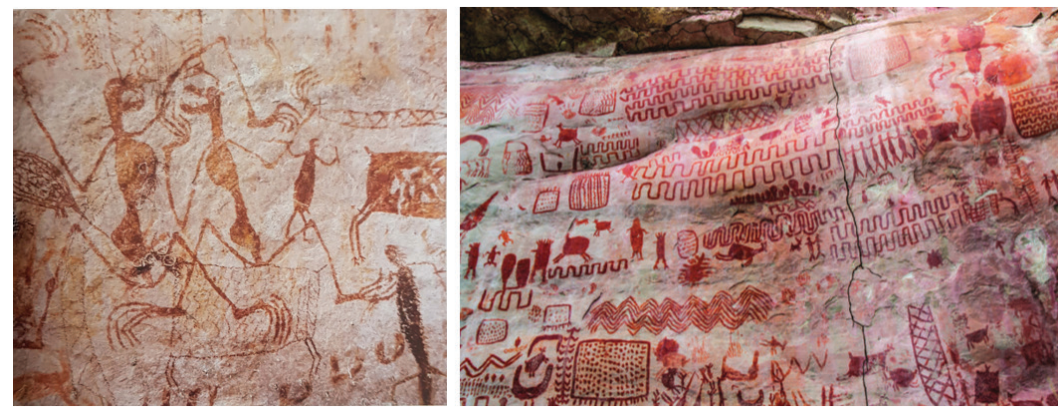

Fuente: Castaño, 2020, p. 535; Urbina y Peña, 2016, p. 14. Nota: Tanto el Chiribiquete como La Lindosa son lugares arqueológicos antiquísimos. Por una parte, los pictogramas más antiguos del Chiribiquete se remontan incluso hasta hace 19.500 años; La Lindosa, por otra parte, hasta 12.000.

9 Cardeña y Beard (1999) realizan un análisis interesante en el que, a partir del acto (como acción y como actuación) chamánico, se evidencia cómo en esta cosmovisión la dicotomía real/irreal se diluye. 
Ahora bien, del hecho de que se afirme la existencia de todas estas entidades no se sigue que los chamanes ${ }^{10}$ no den cuenta del porqué en la vida cotidiana no podemos percibir a estos entes. Para poder explicar ello se recurre al concepto de 'energía' o 'cuerpo energético', ${ }^{11}$ entendido como aquella dimensión substancial que dinamiza (deviene) todas las entidades; algunas de las cuales son físicas y algunas otras no. Así, podemos identificar, cuando menos, dos dimensiones igualmente materiales, energética y física, donde la primera es el fundamento de la segunda. Dice el chamán William Torres (2004a):

Todo hace parte de una inmensa realidad que es indescifrable. Hay una realidad inmediata, a la que consideramos como única realidad, pero que corresponde más bien al modo en que nos hemos habituado a vivir dentro de esa dimensión y a la manera como construimos a nivel cultural y social, tanto que cuando algo se sale de lo habitual se piensa que es irreal. (p. 135).

En este sentido, podemos hablar de dos dimensiones materiales de una misma realidad, donde las entidades específicas

10 «En la lengua Evenk, de Siberia (Rusia), significa "el que sabe" [...] Es un especialista religioso conocido en Colombia como payé (Desana), piache (Orinoquía), máma (Sierra Nevada de Santa Marta), éèmohán (Malibú), jeque (Muisca), jaibaná (Embera), taita (Sibundoy) o kareka (Sierra Nevada del Cocuy)» (Rodríguez Cuenca, 2011, p. 151). Los chamanes suelen entenderse como una persona que tiene la capacidad de establecer vínculos entre esas dimensiones a partir de transformaciones o metamorfosis para, desde allí, realizar acciones que permitan reestablecer el orden cósmico de la naturaleza dictaminado por la ley de origen (tema explicado más adelante). Esas transformaciones las podría realizar por medio de las plantas de saber (o 'enteógenos'), principalmente. Por razones de extensión, no es posible explicar en detalle todos los aspectos fundamentales de esta importante figura de la cosmología abyayalense; sin embargo, el lector puede remitirse al libro de James y Jiménez (eds.) (2004) y Castaño Uribe (2020) para comprender mejor en qué consiste el chamanismo y cuál es su importancia en la cosmovisión indígena, no solo colombiana, sino del resto del mundo.

$11 \quad$ Algo similar podemos encontrar en la cosmovisión indígena japonesa de los siglos IV y V, donde se concebía a ese substrato metafísico del mundo como tama (玉) («poder espiritual») y a cada concreción de ese infinito poder espiritual (este animal, esta deidad, etc.) como kami (神) (Heisig, Kasulis, Maraldo y Bouso, 2016, p. 29). De igual manera, esto lo podemos encontrar en los mayas, para quienes «[...] los dioses eran seres invisibles, pero se manifestaban en seres materiales: astros, plantas, animales, etc., que se convertían en epifanías o manifestaciones de los dioses» (Beorlegui, 2010, p. 95). 
se concretizan gracias a un poder energético infinito y transversal a toda la realidad que este fundamenta. Así, sostener una inmanencia no imposibilita hablar de una trascendencia en un sentido parcial y bajo ciertos matices; es decir, una trascendencia entre ciertas regiones de una misma realidad inmanente. Lo que articularía estas dimensiones en una misma realidad sería, precisamente, la energía, ${ }^{12}$ bien sea manifiesta o no manifiesta. «El más allá no es sino este mismo mundo pero en otra dimensión. Ellos nos ven y nos escuchan mientras que nosotros no los podemos ver y escuchar» (chamán citado por Páramo, 2004, p. 62). Podemos graficarlo así:

Esquema 1. Procesos de concreción y dispersión entre las dimensiones materiales energética y física.

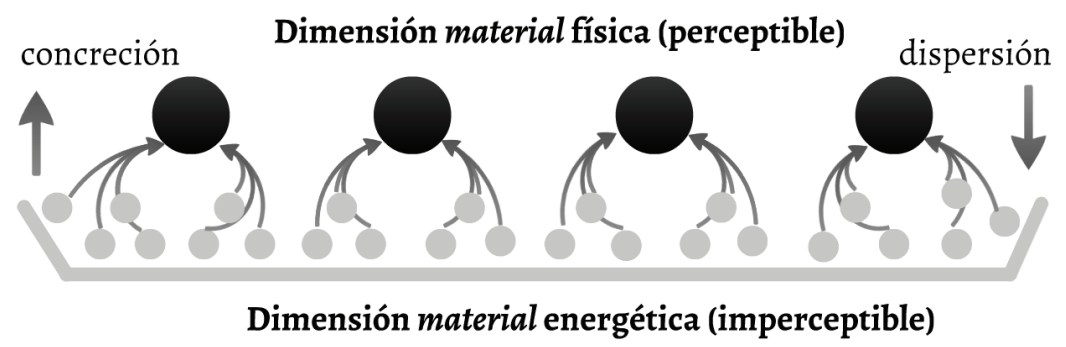

Fuente: elaboración propia.

\begin{abstract}
$12 \quad$ Si bien esta tesis puede resultar escatológica o mística, no debe confundirse el término con tendencias new age sobre esto (Sarrazin, 2012; Fernández, 2009; Arregi, 2011). Como veremos a continuación, a pesar de que se asume este tipo de fundamento, la forma en que este opera se explica de forma estructurada y coherente. A veces, para evitar esas connotaciones se utiliza el término 'espiritual'; sin embargo, es erróneo si lo vemos a la luz del dualismo occidental: la energía también es material. Más bien, 'energía' se refiere aquí a la fuerza vital ínsita de todas las cosas que posteriormente se puede o no concretizar en ciertas entidades físicas concretas. De hecho, curiosamente algo similar (claro está, con sus diferencias), ya había mencionado Confucio (1975) —claro está, según la versión de Zhū Xī-: «iQué amplio y profundo es el poder de las fuerzas sutiles de la naturaleza! Intentamos descubrirlas, pero no las vemos; queremos comprenderlas, pero nos resultan inasequibles; se identifican con la misma esencia de las cosas y no podemos separar la de ellas» (Chung Yunng, XVI, 1-2) y luego amplía: «Estas partículas sutiles e imperceptibles se manifiestan a través de los seres corporales; puesto que existen real y verdaderamente, no pueden dejar de manifestarse de una forma u otra» (XVI, 5).
\end{abstract}


Naturalmente, desde un punto de vista estrictamente metafísico, es perfectamente posible que existan dimensiones que no podemos fácilmente percibir. De hecho, es este uno de los temas actuales de debate en la metafísica contemporánea a partir de la hipótesis de la teoría de cuerdas para proponer una teoría de los campos unificados: el tetradimensionalismo (la posibilidad de la existencia de más de tres dimensiones espacio-temporales) (Ney, 2014). Dicho de forma sencilla: ¿existe solo lo que percibimos o es posible que existan otras entidades, dimensiones, etc. que no necesariamente podemos percibir? Esta pregunta, de hecho, no es más que una moderna reformulación de otra más antigua: «iexisten entidades independientes de la percepción que tengamos de ellas?».

Siendo consecuentes con el pluralismo de entidades, la propuesta acerca de la existencia de múltiples dimensiones permite explicar por qué, a pesar de haber entidades que no podemos experimentar prima faciæ,,$^{13}$ se puede sostener el panrealismo mencionado anteriormente. Gracias a esa multiplicidad de dimensiones se pueden explicar ciertas entidades y hechos que desde una experiencia sensible ordinaria no se pueden explicar. Sin embargo, al estar fundamentada esta dimensión física en una energética, la conexión entre estas no suele plantearse como problema; contrario a lo que pasa en Occidente con el dualismo cartesiano (Descartes, 2014 [Med.], AT, VII, 63 y ss.). En la cosmovisión indígena, la energía puede entenderse como el principio difícilmente perceptible, pero material, que se manifiesta a partir de múltiples dimensiones y entidades. Además, la misma energía sería aquel motor que dinamizaría esas dimensiones para que según su concreción o dispersión se manifieste en algunos cuerpos o entidades específicas. En síntesis: el compromiso ontológico de la cosmología abyayalense se traduce, en término último, en la energía y las múltiples manifestaciones de

$13 \quad$ Es preciso aclarar que con esto no se quiere decir que para los indígenas no haya forma alguna de experimentar esas otras dimensiones energéticas, sino que esas dimensiones no se experimentan en la vida diaria de una forma evidente para el hombre occidental. Para el indígena, los acontecimientos de esta dimensión son reflejo de los hechos de las otras dimensiones, pero solo el chamán puede comprender cabalmente el tipo de conexión entre ambos y qué acción se debe realizar en consecuencia. 
esta en las dimensiones y los diversos cuerpos energéticos. P Así lo explica el indígena Antonio Guzmán (2004):

La energía es el círculo vicioso que unifica el micro y macrocosmos donde el macrocosmos masifica el microcosmos para que puedan suceder las cosas aquí abajo, para que existan los peces, los animales y canten las ranas y los pájaros. (p. 78).

Ahora, en este punto es probable que el lector se pregunte: ¿qué es «micro y macrocosmos» y cómo se articula todo ello de forma ordenada? Lo explicaremos a continuación.

\section{La ley de origen: espacio-tiempo, pedir permiso y pagamento.}

Una vez comprendido el compromiso ontológico de la cosmología abyayalense es necesario ahora abordar de forma general la manera en que esas dimensiones y entidades están articuladas de forma fundacional y armoniosa. ${ }^{14}$ Para comprender ello es crucial entender el concepto de 'ley de origen', así como su modo de operar en el entretejimiento del espacio-tiempo y los diferentes planos espaciales.

El concepto de 'ley de origen' es fundamental en la cosmología abyayalense, ya que permite articular $-\mathrm{o}$, mejor, entretejer - esa basta pluralidad de entidades animadas y dimensiones mencionadas anteriormente. Así lo explica el mama («chamán») Ramón Gil, citado en el Diagnóstico y líneas de acción para las comunidades wiwa de la Sierra Nevada de Santa Marta (departamentos Cesar, Magdalena y Guajira) en el marco del cumplimiento del auto 004 de 2009:

Primero era she, no había tierra, no había agua, no había nada, pero el Mundo siempre existió espiritualmente, todo estaba en pensamiento, entonces en pensamiento se creó seishankua y seinekun para que crearan el mundo y así empezaron a crear el mundo material, las aves, los árboles, los animales pero dijo hay que crear una ley para poder vivir en armonía esa ley es el shembuta [ley de origen]. (Gil citado por: Ministerio del Interior, Organización Wiwa Yugumaiun Bunkuanarrua Tayrona y Organización Delegación Wiwa, 2015, p. 403).

\footnotetext{
$14 \quad$ En este sentido, pasaríamos del estudio de la ontología abyayalense («¿qué existe?») a su metafísica («¿cómo se relacionan esas entidades de forma causal, mereológica, temporal, espacialmente?») (cfr. Hernández, 2020a).
} 
Y justo después se amplía más la explicación de la siguiente manera:

En el mundo espiritual she es un principio de existencia y pensamiento del universo. En este mundo lo material siempre está sostenido por lo espiritual, lo cual a su vez se sustenta en la ley que mantiene el orden de las cosas y el universo, con ella se legisla y se gobierna desde el principio hasta el final de los tiempos. En el inicio de los tiempos los padres espirituales estudiaron la formas de organización espiritual para llegar a materializar el pensamiento, así cuando los mamas realizan consultas se están en comunicación con she con las que buscan tomar decisiones de acuerdo a la ley de origen. (p. 403).

En este orden de ideas, la ley de origen ${ }^{15}$ es un principio cosmológico metafísico según el cual el actuar humano debe regirse conforme al ordenamiento del universo y, por ello, permanecer en esa armonía cósmica. La ley de origen, entonces, es un principio de ordenamiento, no solo metafísico, sino también ético, social y jurídico. Puede resumirse en la armonización, tanto cosmológica como social que dictamina el curso de los hechos y acciones. Tal y como lo muestra Murillo (2013),

Para el restablecimiento del Pensamiento Mayor se debe recurrir a la síntesis del origen, esto implica volver al mito. Aquí se explican dos creaciones principales: la creación del universo a través de la figura de la madre y la creación del mundo material a través de las figuras de Serankwa y Seinekun, a partir de la Sierra Nevada de Santa Marta: "corazón del mundo", los cuales, junto con una gran cantidad de Padres Espirituales, conforman la autoridad tradicional de orden espiritual que en su conjunto estructuran la Ley de Origen y dan soporte simbólico al sistema de las autoridades tradicionales. (p. 31).

Concorde a lo anterior, podemos concluir que la ley de origen es el concepto fundamental de toda la cosmovisión indígena colombiana. Con base en ella se explica la orientación de los acontecimientos cosmológicos, el comportamiento de los animales, el actuar del hombre; en una palabra: la vida. Así lo testimonia el indígena nasa Víctor Hurtado:

15 De nuevo, surge una segunda semejanza con el pensamiento chino, donde el concepto de 'ley del Cielo' (天命) es fundamental en la cosmovisión confuciana $-\mathrm{y}$, en general, la sociedad china- para explicar la adecuación de las acciones morales conforme al orden del cosmos (Confucio, 1975, Chung Yunng, XX, 16-17; XVII, 8-10). 
Cuando hablamos de «cosmovisión» hablamos de la vida que conecta con el universo. Pero ¿Cómo estamos conectados con el universo, con el cosmos? Entonces hablamos de la espiral [...] nosotros estamos conectados, no solo con el espacio, sino [también] con los animales, con los demás habitantes de la naturaleza. Entonces para nosotros esta es la vida; es lo que nos hace permanecer y retornar en el tiempo. (NasaCxhaCxha, 2010, mm. 0:32-0:45; 5:04-5:18).

Ahora bien, para entender la figura de la espiral es importante tener en cuenta que la cosmología abyayalense colombiana está fuertemente influenciada por la cultura maya (la cual migró a diferentes lugares del país). Ejemplo de ello son las comunidades de la Sierra Nevada de Santa Marta (kogui, tairona, arhuac, wiwa, wayúu), gunadule, muisca, u'wa, entre otros. En la cosmología maya (y, en general, en casi toda la cosmología abyayalense), la generación del cosmos proviene de una naturaleza dual (femenino/masculino) de los dioses creadores. ${ }^{16}$ Esto se traduce posteriormente en una cuatripartición del espacio a partir de coordenadas (Norte, Sur, Este, Oeste), que, a su vez, pueden verse en al menos tres planos: cielo, tierra e inframundo (Beorlegui, 2010, p. 95).

Lo importante de esta clasificación es que tiempo y espacio se anidan de forma tal que dicho entretejimiento (acá, evidenciado en la espiral) ${ }^{17}$ una vez es constituido es inseparable y, sin embargo, siempre está en construcción (devenir) constante. Espacio y tiempo son, para los indígenas, inseparables; están entretejidos, hacen parte de una misma realidad. Así se podría graficar:

$16 \quad$ Este aspecto se puede evidenciar de sobremanera en el Ometeotl de los nahuas (León-Portilla, 2017, pp. 201 y ss.; Hernández, 2019, pp. 263264) y en el Pachacamac de los incas (Estermann, 2009, pp. 277 y ss.).

17 En realidad, la clasificación puede ser mucho más compleja, proponiendo hasta 11 subniveles en uno o varios planos. Para un mayor análisis, véase: Díaz (ed.) (2015). Si bien a continuación se muestra el entrelazamiento desde la espiral (la figura más estándar) (Giraldo, 2010), es preciso tener en cuenta que no es la única forma de estructurar el cosmos. Por ejemplo, como lo muestra Reichel-Dolmatoff (1997), los desana proponen una estructura hexagonal (pp. 150 y ss.); de igual manera, en algunos tejidos de La Lindosa pueden verse estructuras más cercanas a unas redes (Urbina y Peña, 2016, pp. 31-32). Incluso, como se evidencia en el video de la entrevista al indígena Víctor Hurtado (NasaCxhaCxha, 2010), la espiral podría representarse de múltiples maneras... 
Esquema 2. Articulación de espacio y tiempo por la ley de origen en la cosmología abyayalense.

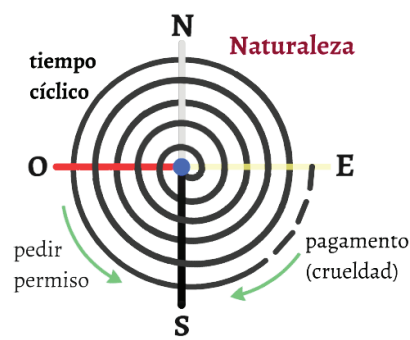

= ley de origen

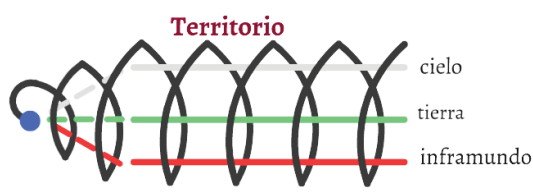

Fuente: elaboración propia. Obsérvese detalladamente que en la espiral frontal tiempo y espacio se entrelazan, mientras que en la que está de perfil el tiempo acoge los tres planos dentro de un mismo campo común.

Ahora bien, dicho entrelazamiento suele denominarse como 'tahuanintisuyu' (espacio-tiempo). Es gracias a ese entrelazamiento (chacana) que se genera un vínculo entre lo macro y lo microcósmico: el territorio (Torres, 2004b, p. 24). Gracias a ese entrelazamiento espiralado de espacio y tiempo se armonizan las dimensiones y entidades como un cosmos («orden»:

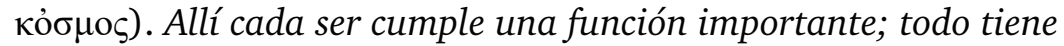
un sentido, todo está entrelazado: los dioses (en especial, el Sol y la Tierra), quienes crean el mundo a partir de la dualidad engendradora (dios seminal, diosa madre), ${ }^{18}$ los elementos (agua, tierra, fuego, aire), como la cuatripartición (las coordenadas de la espiral) derivada de esa dualidad y que vivifica todo cuerpo físico (Castaño, 2020, p. 111); las piedras, el ser más antiguo en esta Tierra, axis mundi y centro de origen (p. 120); las plantas (sobre todo, las enteógenas), el ser donde se condensa y se preserva el saber de la ley de origen (De la Garza, 2012; Castaño, 2020, pp. 204 y ss.); y los animales, aquellos con quienes se tienen vínculos para proteger a la naturaleza; $y$, como lo indica el profesor e indígena mayor ${ }^{19}$ Abadio Green: «Nosotros, los seres humanos, somos lo último de la creación» (Green, 2018, mm. 33:35).

$18 \quad$ Este aspecto se puede evidenciar de sobremanera en el Ometeotl de los nahuas (León-Portilla, 2017, pp. 201 y ss.; Hernández, 2019, pp. 263264) y en el Pachacamac de los incas (Estermann, 2009, pp. 277 y ss.).

19 En el contexto indígena, se llama «mayor» en algunas comunidades al maestro espiritual de la comunidad. Podría tomarse como sinónimo de 'chamán'. 
Contrario al pensamiento del hombre occidental, el indígena no ve a la naturaleza como un depósito que hay que explotar; ${ }^{20}$ al contrario, al tener un vínculo próximo con el resto de los seres, considera una responsabilidad el cuidado del territorio. Por ello, son fundamentales los dos mandamientos de la ley de origen: «pedir permiso» y el « hacer pagamento». Así lo explica Torres (2004b):

Este mundo es Nuestra Madre. Por eso hay que protegerlo y mantenerlo fértil. Nosotros los hijos de Sekuanya, La Gente Jaguar, tenemos que cuidarlo, haciendo siempre los ceremoniales, los cantos y los bailes, para no olvidar nunca la Ley de La Madre, nuestro Mugi Gabá. (p. 38).

La ley de origen — si bien tiene una connotación cosmológica abstracta- se traduce en términos prácticos para el hombre en el cuidado de la naturaleza. La ley o mandato es mantener la armonía del cosmos a partir del pedir permiso y del pagamento. Mientras el hombre occidental toma el fruto del árbol arbitrariamente; el indígena, reconociendo al árbol como un ser vivo y animado, pide primero permiso antes de tomar el fruto. Igual sucede con el tratamiento del agua, la tierra, las piedras y los animales. Todos son seres vivos y animados, hacen parte del tejido de la naturaleza y, por lo mismo, merecen respeto.

Pero, más aún, no basta para el indígena tener respeto por los demás seres, sino que es necesario realizar prácticas especiales en sitios sagrados para que esa armonía permanezca. En ello consiste el pagamento: volver a los mitos, volver al lugar sagrado (duashagaka) donde todo se originó para reencontrarse con el fundamento de la existencia de todo. Dice el mamo Lorenzo Izquierdo:

20 Téngase en cuenta que esta reducción de la naturaleza a un depósito que hay que explotar solo aparece en Occidente en los inicios de la Modernidad con Bacon (1985 [NO], I, 11) con la idea de que, basado en la Biblia, es Dios quien nos ha dado el dominio sobre el resto de la naturaleza (I, 26, 28 y ss.). Previo a esto, en la tradición griega la púoıs se veía como objeto de contemplación (Heidegger, 1997, pp. 122 y ss.). De igual manera, en el Medioevo la naturaleza solía verse como objeto de respeto por ser una creación de Dios (Aquinatis, 1951 [ST], I, q. 103, aa. 3, 8). 
[...] para que vuelva a estar en equilibrio esa naturaleza tenemos que hacer pagamento todo el tiempo, porque eso es la función, la vibración de la madre tierra, cuando hacemos los pagamentos. (Bunkuaneyuman Comunicaciones, 2020, mm. 1:03-1:15).

\section{Y luego amplía el líder kankuamo Daniel Maestre:}

Nosotros no podemos hablar de sitios sagrados, sino de áreas sagradas, que son como los lugares especiales que dejaron los padres espirituales para armonizar el universo con funciones bien específicas. [...] Y en últimas, nosotros hemos llegado a la conclusión [de] que esos lugares son ecosistemas endémicos o únicos que sirven para armonizar el medio ambiente. (1:16-1:32; 1:50-2:03).

Resumiendo, la ley de origen es, pues, tanto el principio cosmológico según el cual se entretejen espaciotemporalmente todas las dimensiones, entidades y fenómenos; así como la norma de mantener ese ordenamiento a partir del respeto a los demás seres y del retorno — también espaciotemporal- a los lugares sagrados para reencontrarse con el mito y con el origen de todas cosas. ${ }^{21}$

\section{La visión holográfica: micro y macrocosmos}

Ahora bien, todo esto (la ley de origen: el pedir permiso y el pagamento) cobra sentido a partir de un último principio:

\footnotetext{
$21 \quad$ Actualmente se está realizando el proyecto multiplataforma El buen vivir, en el que Comisión Nacional de Comunicación de los Pueblos Indígenas (CONCIP) «[...] invita a descubrir una parte de la inmensa riqueza cultural de nosotros, los pueblos originarios de Colombia. Queremos compartir nuestros modelos de solución a distintos problemas asociados a la modernidad. No se trata de un simple recetario sino de ideales y prácticas inspiradas por distintos enfoques de economía solidaria, soberanía alimentaria, derechos de la naturaleza, protección de la biodiversidad, defensa del territorio, buen gobierno, comunicación espiritual, resolución de conflictos y convivencia ciudadana en la diferencia cultural» (CONCIP, 2020, § 1). Se le recomienda al lector la exploración de estos materiales.
} 
la visión holográfica. ${ }^{22}$ Esta se entiende como el isomorfismo entre aquello que sucede a nivel universal y lo que acontece a nivel particular. Para los indígenas colombianos, lo macro y lo micro están articulados por la energía y la ley de origen, de allí la necesidad de realizar el pagamento (las prácticas especiales que mencionamos anteriormente).

Siguiendo al indígena tukano Antonio Guzmán (2004),

Macrocosmos es el mismo universo donde están los planetas y las estrellas. Para nosotros, todas las estrellas tienen su significado, si algo sucede es porque una estrella lo anunció, determinadas estrellas siempre anuncian la lluvia. [...] Entonces la cuestión del universo no es por azar, como dicen muchos en Occidente [...] todas las cosas tienen ese sentido común, como ese aviso, esa señal. Sabemos cuándo se deben preparar la mandioca y el casabe, cuándo comer. Todo el universo es hermoso. El microcosmos es en donde estamos, la selva, los animales, la caza, la siembra, las chagras, hasta lo más pequeño. (p. 77).

Precisamente esa sinergia entre lo macro y lo micro se refleja, entre otras muchas cosas, en la construcción de la maloka. Debido a esa articulación es que la generación y retorno del origen se convierten ley: natural, por una parte, en tanto generadora de todas las cosas; ética, por la otra, en tanto que el chamán - y en general, todos los miembros de la comunidad a través de él- vuelven al lugar sagrado para reencontrarse con el mito y reestablecer ese puente entre lo micro y lo macrocósmico. Dice Urbina (2004):

[...] Pero también es un modelo sincrónico de lo que es el cosmos: si se detiene el movimiento (proceso) del cosmos, si se hace un corte sincrónico, ese cosmos puede tener la forma de una maloka. [...] Supongamos que una persona indígena no ha recibido esa enseñanza completa, pero entra en la maloka, la ve, la observa. Él puede, estableciendo relaciones entre las diferentes partes de la maloka, volver a reconocer el discurso básico, su sentido fundamental. (p. 102).

22 De nuevo, vuelve a surgir un punto común con Japón. Como lo indican Heisig, Kasulis, Maraldo y Bouso (2016): «[...] un enfoque "holográfico" ve un "todo inscrito" en cada una de sus partes. Esto solo es posible si las partes están relacionadas interna, en lugar de externamente. El pensamiento holográfico, aunque no esté del todo ausente en la tradición filosófica occidental, es la forma del pensamiento básico de la japonesa» (pp. 49-50). Este principio ya se encontraba en el confucianismo (Confucio, 1975, Chung Yung, XXVI, 6 y ss.) y daoísmo chino (Laozi, 2017, c. XVI), aunque el culmen de su desarrollo se alcanzó con el budismo huayen (Cleary, 1983). 
Como bien lo indica Urbina, la maloka es un fiel reflejo del orden cósmico (vid. supra esquema 2): se construye primero indicando los cuatro puntos cardinales y conforme a ellos se elabora un tejido en forma de espiral. Por ello, la espiral no se entiende como una idea abstracta, lejana o como una ficción, sino que para ellos es el fiel reflejo del orden de todas las cosas, tanto a nivel macro (los planetas, las constelaciones, la Tierra, etc.) como a nivel micro: la corona de la cabeza, el tejido de los sombreros, de las mochilas, las huellas de los dedos de las manos y pies, los animales: en su frente y espinazo, los árboles, el crecimiento de las plantas, etc. (NasaCxhaCxha, 2010, mm. 4:08-5:00). Esto también se evidencia en la orfebrería y en los pictogramas:

Ilustración 2. Ejemplos de espirales en orfebrería indígena colombiana.
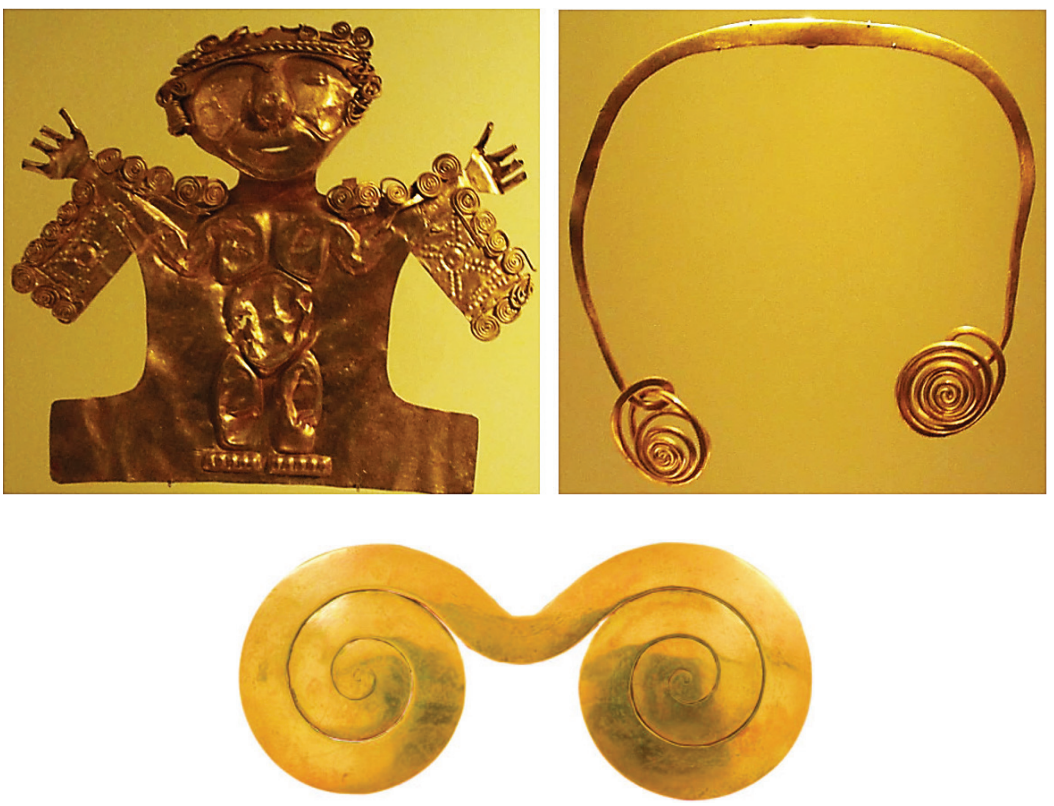

Fuente: imágenes 1 y 2: Museo del oro, Bogotá, Colombia; fotografía: Consuelo Pabón Alvarado. Imagen 3: (Museo del oro del arte precolombino, Museo del oro - Bogotá, 2005, p. 21). 
Ilustración 3. Ejemplo del cosmos en espiral en huella ubicada en el Chiribiquete.

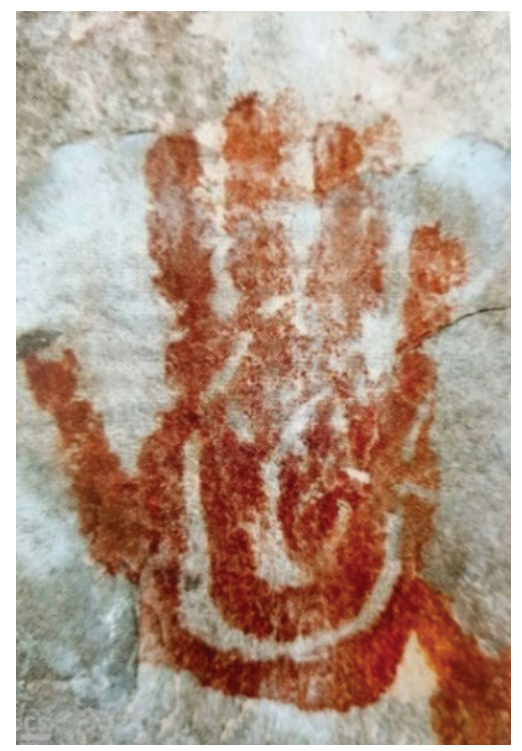

Fuente: Castaño, 2020, p. 536.

A nivel macrocósmico, fácilmente podemos tener como ejemplo de la espiral en la vía láctea. Pero, más aún, la creación, como se indicó en la sección anterior, se entiende como síntesis entre lo masculino y lo femenino. Lo masculino suele simbolizarse con el Sol y lo femenino con la Tierra. Cada uno se manifiesta en dos animales específicos: el jaguar (el Sol) y la boa (la Tierra). Precisamente, «[...] se considera , además, que los eclipses son los intentos de uturuncu por devorar a Sol y Luna. Uturuncu constituye la potencia primordial de Uiracucha pachayachachic (el creador, sabio y cuidador del Cosmos)» (Torres, 2004b, p. 11). Consecuentemente, esto se puede evidenciar en las estrellas (la escritura de los dioses, para los uitoto), donde se puede ver en las constelaciones al jaguar inseminando la Tierra (véase ilustración 4): 
Ilustración 4. Constelaciones en las que se evidencia al jaguar inseminando la Tierra.

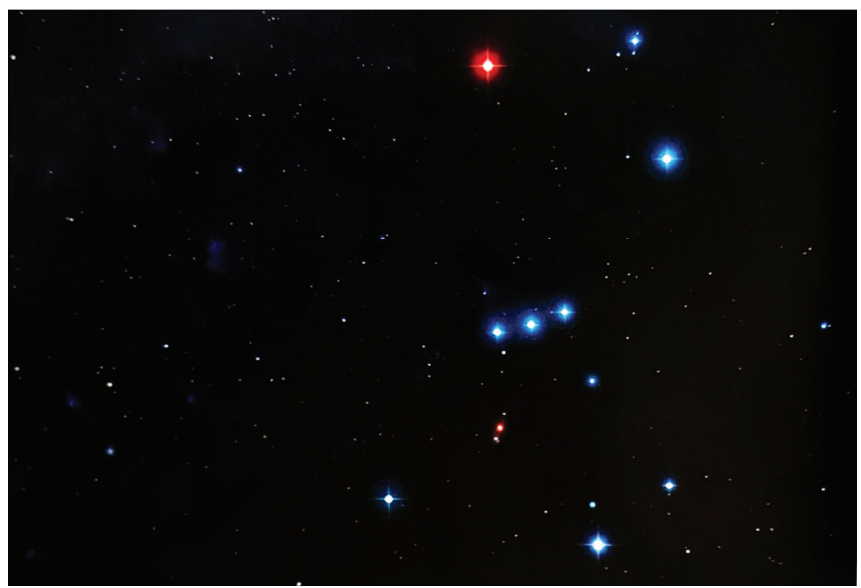

Fuente: Castaño, 2020, p. 466. Nota: Dice Castaño (2020) en la descripción de la fotografía: «Las cuatro estrellas principales de los extremos (Betelqeuse, Saiph, Rigel y Bellatrix) son las patas desplegadas del jaguar en su salto felino. El falo son las tres del centro (Alnitak, Alnilam y Mintaka) y debajo de estas, el semen del jaguar (nebulosa M2), mientras que su cola es como un gran arco que va por el costado Bellatrix-Rigel» (p. 466).

De la misma manera, como lo muestra Torres (2004b), para los indígenas (no solo los ingas e incas, sino en general, casi todo el pensamiento amerindio) el jaguar es un símbolo fundamental en su cosmovisión. Por ejemplo, como él lo indica, esa figura también se puede evidenciar en la construcción de la ciudad de Cuzco (véase ilustración 5):

Ilustración 5. Representación del jaguar en la estructura de la ciudad de Cuzco.

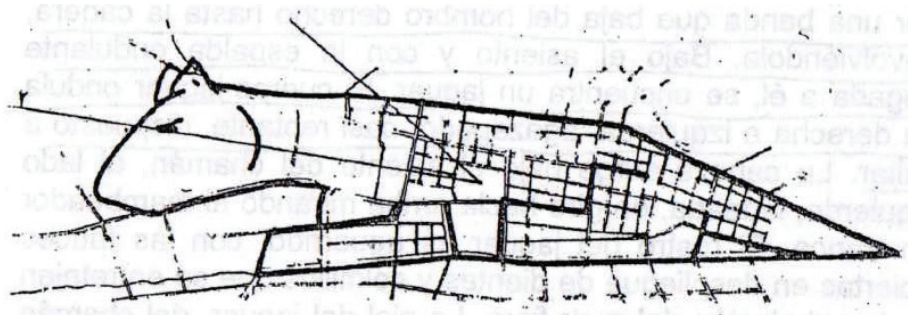

Fuente: Torres, 2004, p. 11. 
La construcción de la maloka, la orfebrería y los pictogramas (a nivel micro), así como las constelaciones (a nivel macro) son tan solo un reflejo de la interconexión que hay entre lo universal y lo particular. Para los indígenas, las distinciones que el occidental realiza entre lo macro y lo micro no tienen cabida; una vez más, todo hace parte de una misma realidad entrelazada por un mismo principio y fundamento: la energía orientada por la ley de origen. Por esto, quizás el mayor ejemplo lo podemos encontrar en el origen (la ley de origen) de la vida de cada uno de nosotros. Así lo explica el profesor y mayor indígena guna dule Abadio Green (2018):

Cuando comenzó la danza de la espiral, vi a los abuelos y abuelas danzando. Danzaban en una espiral hasta que cuando empezó a calentarse, nació el primer ser de esa relación del óvulo y el espermatozoide. El primer ser fue el agua. Por eso ustedes, mujeres, madres, abuelas; ustedes solamente, las mujeres, crean el agua [...] Nosotros, todos los seres nacimos de esa agua: es el líquido amniótico [...] Y ustedes tienen una matriz que está ligada con la madre Tierra, los hombres no. [...] Su cuerpo está dotado de esa relación; eso fue lo que me dijeron las abuelas. (mm. 27:12-28:40).

En este sentido, la vida misma no es más que una reiteración perpetua de esos ciclos naturales que, como lo indica el profesor Green, son manifestación de una armonía cósmica: la ley de origen. Por eso, esa estructura explicada anteriormente (vid. supra esquema 2) fundamento metafísico y ético no representa -en el sentido mencionado en la sección 1 de este texto- el orden de las cosas, sino que se manifiesta, es en todas las cosas. La ley de origen no solo es el puente o la articulación de micro y macrocosmos; es en el micro y macrocosmos. Por eso, ambos se reflejan o proyectan (como un holograma) a partir de relaciones internas, ya que todo proviene de un mismo origen/ fundamento que entrelaza todo cuanto existe. Así lo amplía el mismo Abadio Green:

Todos venimos de un mundo espiral. Todos, porque todo, cuando nos hicieron a nosotros, cuando estuvimos en el vientre de nuestras madres, antes de que fuéramos seres con músculo, éramos agua, fuimos agua. Y esa agua danzó, esa agua entendió el tiempo originario, que es el tiempo espiral. Nuestro ADN es espiral, nuestra cabellera es espiral, nuestras huellas son espiral, nuestros ojos son espiral; todo es espiral: nosotros los seres humanos. El águila tiene 
que cazar en espiral porque si no, se muere. Todos los animales, toda la naturaleza es espiral. Cuando una semilla está saliendo de la madre tierra, es espiral; no sale directo. Toda planta sale danzando a la madre Tierra. (38:55-40:03).

\section{Conclusiones}

Hasta el momento hemos reconocido los siguientes elementos claves para la comprensión de la cosmología abyayalense: primero, que es inmanente y monista; y, a la vez, múltiple en su interior. Segundo, que se reconocen múltiples dimensiones, identificables con dos tipos de corporalidades materiales: el cuerpo físico y el cuerpo energético (el segundo es el fundamento del primero, y el primero es concreción del segundo). Tercero, que el fundamento metafísico es la ley de origen, la cual se desenvuelve en el entrelazamiento (chacamac) espacio-temporal que permite articular esas dimensiones múltiples de forma armónica; y que, además, esa ley de origen se traduce en el cuidado del territorio: el pedir permiso y el pagamento. Y cuarto, que esa armonía se refleja en un isomorfismo entre macro y microcosmos, lo cual refuerza la idea de generación y retorno al origen basándose en que, como se indicó antes, todo está conectado por esa ley. Esos cuatro principios filosóficos fundamentan no solo sus mitos y prácticas, sino su vida.

En este punto es difícil hablar aquí de «conclusiones»; más bien, ante las elucidaciones y precisiones conceptuales de los principios metafísicos de la cosmología abyayalense colombiana lo que se nos presenta es el reto de desarrollar con mayor detalle y precisión cada uno de los problemas metafísicos presentes en esos principios cosmológicos (el problema del tetradimensionalismo, el modelo fundacional expuesto en la espiral, el espacio y el tiempo como codependientes, la visión holográfica entre micro y macrocosmos, la concepción ética del pedir permiso y el pagamento, etc.), así como sus relaciones interculturales con otras filosofías (la griega, la china y la japonesa). Considérese que este texto es, pues, una invitación a la investigación filosófica del pensamiento indígena de nuestros territorios. Quizás, algo de lo que aprendamos de ellos no solo 
amplíe nuestra perspectiva filosófica acerca de cómo construir modelos de mundo menos dualistas, sino también en asumir nuevas prácticas filosóficas y mejores modos de relacionarnos con el otro y con la naturaleza; sobre todo, con esta última.

\section{Referencias}

AQUINATIS, s. T. Summa theologiæ. Pars I. Madrid: Biblioteca de Autores Cristianos, 1951.

BACON, F. La gran restauración. Trad. Miguel A. Granada. Madrid: Alianza, 1985.

BEORLEGUI, C. «El pensamiento indígena en la América pre-colombina». En: Historia del pensamiento filosófico latinoamericano: una búsqueda incesante de la identidad (pp. 81-112). Bilbao: Universidad de Deusto, 2010.

BERTO, F. y PLEBANY, M. Ontology and Metaontology. London: Bloomsbury, 2015.

CARDEÑA, E. y BEARD, J. Artificios verídicos: chamanismo, actuación y realidad, 58 (1), 1999, 99-112. Recuperado de: https://cdigital. uv.mx/handle/123456789/4523.

CASTAÑO URIBE, C. Chiribiquete: la maloka cósmica de los hombres jaguar. Bogotá: Sura, 2020.

CLEARY, T. Entry Into the Inconceivable: An Introduction to Hua-yen Buddhism. Honolulu: University of Hawaii Press, 1983.

Comisión Nacional de Comunicación de los Pueblos Indígenas (CONCIP). El buen vivir (proyecto de recolección documental multiplataforma). 2020. Recuperado de: http://elbuenvivir.co.

DE LA GARZA, M. Los mayas. Antiguas y nuevas palabras sobre el origen. En: MONJARÁS-RUIZ, J. (Ed.). Mitos cosmogónicos del México indígena (pp. 15-86). México: Instituto Nacional de Antropología e Historia, 1987.

DE LA GARZA, M. Sueño y éxtasis: visión chamánica de los nahuas y mayas. México: Universidad Nacional Autónoma de México, 2012.

DESCARTES, R. Meditaciones acerca de la Filosofía Primera. Trad. $1^{\mathrm{a}}$ y $2^{\mathrm{a}}$ ed. Jorge Aurelio Díaz. Edición trilingüe. Bogotá: Universidad Nacional de Colombia, 2014.

DÍAZ, A. (Ed.). Cielos e inframundos: una revisión de las cosmologías mesoamericanas. México: Universidad Nacional Autónoma de México, 2015.

ESTERMANN, J. Filosofía andina: sabiduría indígena para un nuevo mundo. La Paz: Instituto Superior Ecuménico Andino de Teología, 2009. 
GIRALDO JARAMILLO, N. Camino en espiral Yo"sa Ingunu territorio sagrado y autoridades tradicionales en la comunidad indígena IKU de la Sierra Nevada de Santa Marta. Manizales: Universidad de Caldas, 2010.

GREEN, A. Cosmovisión de nuestros ancestros. En: Seminario tríptico. Universidad EAFIT, Medellín. 2018. Recuperado de: https:// www.youtube.com/watch? $v=$ itggkiVs0ZM\&t=20s.

GUZMÁN, A. El chamán, el jaguar, la selva. En: JAMES, A. J. y JIMENEZ, D. A. (Eds.). Chamanismo: el otro hombre, la otra selva, el otro mundo. Bogotá: Instituto Colombiano de Antropología e Historia, 2004, pp. 69-82.

HADOT, P. La filosofía como forma de vida: conversaciones con Jannie Carlier y Arnold I. Davidson. Trad. María Cucurella Miquel. Barcelona: Alpha Decay, 2009.

HEIDEGGER, M. La pregunta por la técnica. En: Filosofía, ciencia y técnica. Trad. Jorge Soler y Jorge Acevedo. Santiago de Chile: Editorial Universitaria, 1997, pp. 111-148.

HEISIG, J. W. et al. (eds.). La filosofía japonesa en sus textos. Barcelona: Herder, 2016.

HERNÁNDEZ RODRÍGUEZ, J. C. El Ometeotl: la dualidad como fundamento metafísico trascendental. Perseitas, 7 (2), 2019, pp. 248-273.

HERNÁNDEZ RODRÍGUEZ, J. ¿En qué debería consistir el conocimiento metafísico contemporáneo? Scientia in Verba Magazine, 6(1), 2020a, pp. 136-148.

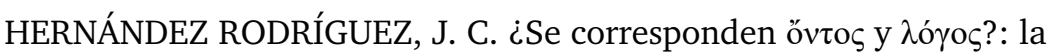
pregunta fundamental de la metafísica griega. Eidos, 33, 2020b (en edición).

JAEGER, W. Paideia: los ideales de la cultura griega. Trad. Joaquín Xirau y Wenceslao Roces. México: Fondo de la Cultura Económica, 2001.

JAMES, A. J. y JIMÉNEZ, D. A. (eds.). Chamanismo: el otro hombre, la otra selva, el otro mundo. Bogotá: Instituto Colombiano de Antropología e Historia, 2004.

JAMES, J. A. Introducción. En: JAMES, A. J. y JIMÉNEZ, D. A. (eds.). Chamanismo: el otro hombre, la otra selva, el otro mundo. Bogotá: Instituto Colombiano de Antropología e Historia, 2004, pp. 11-38.

LAOZI. Dao De Jing o Livro do Tao. Edición bilingüe portugués-chino. Trad. Chiu Yi Chih. São Paulo: Mantra, 2017.

LENKERSDORF, C. Cosmovisión maya. México: Centro de Estudios Antropológicos, Científicos, Artísticos, Tradicionales y Lingüísticos "CeAcatl", 2003.

LENKERSDORF, C. Filosofar en clave tojolabal. México: Centro de Estudios Antropológicos, Científicos, Artísticos, Tradicionales y Lingüísticos "CeAcatl”, 2005. 
LEÓN-PORTILLA, M. La filosofía náhuatl estudiada en sus fuentes. México: UNAM, 2017.

LYDELL, H. G.; SCOTT, R. Greek-English Lexicon. Clarendon: Oxford University Press, 1996. Retrieved from: http://www.perseus.tufts. edu/hopper/resolveform?redirect $=$ true.

MAFFIE, J. Aztec Philosophy: Understanding a World in Motion. Colorado: University of Colorado Press, 2014.

MAGOJA, E. E. Sócrates: entre filosofía y chamanismo. Stylos, 27 (27), 2018, 143-155.

MARIÁTEGUI, J. C. El factor religioso. En: 7 ensayos de interpretación de la realidad peruana. Caracas: Fundación Biblioteca Ayacucho, 2007, pp. 134-161.

MATA GAVIDIA, J. Existencia y perduración en el Popol-vuh. Guatemala: Imprenta Universitaria, 1950.

MORGAN, K. Myth and Philosophy from de Presocratics to Plato. Cambridge: Cambridge University Press, 2004.

MURILLO ESCOBAR, D. R. Ley de origen y legislación en Colombia: contraposición, intereses y contradicciones entre los pueblos indígenas y el Estado, en materia de explotación de recursos naturales en la Sierra Nevada de Santa Marta (tesis de pregrado en politología). Universidad Nacional de Colombia, Bogotá, 2013.

Museo Chileno del Arte Precolombino y Museo del Oro - Bogotá. Oro de Colombia: chamanismo y orfebrería (exposición). Santiago de Chile: Museo Chileno del Arte Precolombino, 2005.

NasaCxhaCxha. Cosmovisión nasa (archivo de video). (2010, 1 de diciembre) Recuperado de: https://www.youtube.com/ watch? $\mathrm{v}=$ BjzRmpYM-gs.

NEY, A. Chapter 6: Persistence. In: Metaphysics: An Introduction. New York: Routledge, 2014, pp. 170-189.

NISHITANI, K. La religión y la nada. Trad. Raquel Bouso. Madrid: Siruela, 1999.

OCHOA ABAURRE, J. C. Mito y chamanismo: el mito de la tierra sin mal en los tupí-cocama de la Amazonía peruana (tesis doctoral). Universidad de Barcelona, Barcelona, 2002.

PABÓN, M. C. Estética de la crueldad: América cruel. Texto y Contexto, 72 (1), 1993, pp. 74-97.

PABÓN, M. C. América cruel: una aproximación al doble. Nova et Vetera, 46 (1), 2002, pp. 6-17.

PALACIOS LIBERATO, L. Una investigación acerca de historia de la filosofía en la civilización andina prehispánica. Perseitas, 7 (2), 2019, pp. 274-298. 
PÁRAMO, G. Lógica paraconsistente y el mito chamánico. En: JAMES, A. J.; JIMENEZ, D. A. (eds.). Chamanismo: el otro hombre, la otra selva, el otro mundo. Bogotá: Instituto Colombiano de Antropología e Historia, 2004, pp. 39-68.

QUINE, W. v. O. Acerca de lo que hay. En: Desde un punto de vista lógico. Trad. Manuel Sacristán. Barcelona: Orbis, 1984a, pp. 25-48.

REICHEL-DOLMATOFF, G. Cristales de roca de los chamanes Desana y el universo hexagonal. En: Chamanes de la selva pluvial. Trad. E. Sánchez. Guildford: Themis Books, 1997, pp. 149-162.

REICHEL-DOLMATOFF, G. La cosmovisión chamánica. En: Orfebrería y chamanismo. Bogotá: Banco de la República-Villegas Editores, 2005, pp. 23-31.

RODRÍGUEZ CUENCUA, J. V. Cosmovisión, chamanismo y ritualidad en el mundo prehispánico de Colombia. Esplendor, ocaso y renacimiento. Maguaré, 25 (2), 2011, pp. 145-195.

SARRAZIN, J.-P. New Age en Colombia y la búsqueda de la espiritualidad indígena. Revista colombiana de antropología, 48 (2), 2012, 139-162.

SCHULTES, R. E; HOFMANN, A.; RÄLSCH, C. Plantas de los dioses: las fuerzas mágicas de las plantas alucinógenas. México: Fondo de Cultura Económica, 2000.

SIDER, T.; HAWTHORNE, J.; ZIMMERMAN, D. W. (ed.). Contemporary Debates in Metaphysics. Singapore: Blackwell Publishing, 2008.

TORRES, W. El chamán, el tigre y el cuerpo sin órganos. En: JAMES, A. J. y JIMÉNEZ, D. A. (eds.). Chamanismo: el otro hombre, la otra selva, el otro mundo. Bogotá: Instituto Colombiano de Antropología e Historia, 2004a, pp. 125-149.

TORRES, W. Uturuncu Runa: historias de la gente jaguar. Bogotá: Ediciones Zahir, 2004b.

URBINA, F. Chamanismo y pensamiento abyayalense. En: JAMES, A. J. y JIMÉNEZ, D. A. (Eds.). Chamanismo: el otro hombre, la otra selva, el otro mundo (pp. 83-124). Bogotá: Instituto Colombiano de Antropología e Historia, 2004.

URBINA, F. (ed.). Palabras del origen: breve compendio de la mitología de los uitotos. Bogotá: Ministerio de Cultura, 2010.

URBINA, F. y PEÑA, J. E. Perros de guerra, caballos, vacunos y otros temas en el arte rupestre de la Serranía de La Lindosa (Río Guayabero, Guaviare, Colombia): Una conversación. Ensayos. Historia y teoría del arte, 20 (31), 2016, pp. 7-37.

YÁÑEZ DEL POZO, J. Yanantin: la filosofía dialógica intercultural del Manuscrito de Huarochiri. Quito: Abya-Yala, 2002.

ZELLER, E. Fundamentos de la filosofía griega. Trad. Alfredo Llanos. Buenos Aires: Ediciones Siglo XX, 1968. 